JEL Classification: C11, C18

Keywords: natural condition of control, Bayesian estimation, DSGE model, model adaptability

\title{
A Note on the Role of the Natural Condition of Control in the Estimation of DSGE Models
}

\author{
Martin FUKAČ - Federal Reserve Bank of Kansas City (martin.fukac@kc.frb.org) \\ (corresponding author) ${ }^{*}$ \\ Vladimír HAVLENA - Czech Technical University in Prague (ČVUT) and Honeywell \\ (vladimir.havlena@honeywell.com)
}

\section{Abstract}

This paper is written by authors from the technical and economic fields who are motivated to find a common language and views on the problem of the optimal use of information in model estimation. The center of our interest is the natural condition of controla common assumption in Bayesian estimation in the technical sciences, and one which may be violated in economic applications. In estimating dynamic stochastic general equilibrium (DSGE) models, typically only a subset of endogenous variables is treated as measured even if additional data sets are available. The natural condition of control dictates the exploitation of all available information, which improves model adaptability and estimate efficiency. We illustrate our points on a basic RBC model.

\section{Introduction}

Since the seminal paper by Peterka (1981), it has been well understood in the technical sciences that on the way from the Bayesian formula to the standard recursive least square method for ARX model estimation or Kalman filter estimation, several assumptions about the information contained in the observed input and output variables must be adopted. While such assumptions are well justified and easy to interpret in technical applications such as LQG observer-based state feedback or adaptive control, they may be violated in some other areas such as economics.

Our attention is focused on the natural condition of control (henceforth the NCC or condition). We would like to stimulate a discussion on the proper use of the information available to econometricians and on the adaptation of theoretical model concepts to particular estimation algorithms. We review the development of model estimation from a conceptual Bayesian solution - resulting in a generic functional recursion on conditioned probability density functions (c.p.d.f.) - to the famous Kalman filter equations. We demonstrate the loss of optimality in the case where the assumptions used for the development of the standard Kalman filter are not satisfied.

The natural condition of control is an assumption made in the control system literature that simplifies the algorithm for the optimal estimation of unknown variables such as parameters or state (latent) variables using the Kalman Filter. The condition says that if an external observer (econometrician/statistician) simultaneously observes and controls the system, then his control decisions, if optimal, do not provide any additional information about the state of the system, and vice versa.

\footnotetext{
* The views expressed in this paper are those of the authors and do not necessarily reflect those of the Federal Reserve Bank of Kansas City or the Federal Reserve System.
} 
The violation of NCC is difficult to detect in the data, because the problem is different from that of model misspecification, which manifests itself in residuals, shock estimates, or inconsistent, model-implied expectations. But if econometricians and economic agents with significant market power objectively know that the condition does not hold (i.e., there are observed control variables that are not explicitly includeed in their models when they should be and thus the NCC is violated), the NCC entitles them to use that knowledge in their favor.

In contrast to many economic applications, the NCC is a credible assumption in the technical sciences. The observer and controller are one person, the system under his control is well identified, and he uses algorithms that lead to optimal estimation and decisions. On the other hand, in economic applications it is almost always difficult to argue that the condition holds, because the observer (econometrician) is almost always different from the controller. The econometrician observes the real-time decisions (about tax revenues, production, consumption or prices) with a substantial time delay. Natural candidates for the controller-observer in economics are policy institutions such as monetary or fiscal authorities, which observe the markets' behavior and, most importantly, have effective tools to influence them. The NCC does not apply to economic agents in perfectly competitive markets because their size prevents their behavior from impacting on the aggregate markets. The condition may apply to the abstract concept of a representative agent (which we do not explore here) or agents with significant market power, such as policy institutions.

We review the condition's validity for the estimation of dynamic stochastic general equilibrium (DSGE) models. We choose them because they have become the norm for optimal policy and decision analysis in policy institutions. At the same time, they are exactly the class of economic models for which the NCC is the most relevant because they capture optimal decisions.

There are two direct implications of the NCC for DSGE models. First, we can improve the efficiency of our estimates. Second, because the observed control variable is a result of optimal decisions in these models, we can use that variable to infer the encoded underlying information to improve our own knowledge about the modeled system. The performance of our own model can benefit if the underlying model of the particular optimal decision is of better quality than ours.

We show that the choice of observable variables matters. There are many decision variables that are implicit in DSGE models, but the variables have direct observable counterparts such as labor income, capital income, or all kinds of fees or tax revenues. For model dynamics, they are of second-order importance because they do not carry any extra information for the aggregate dynamics, since output, prices, and interest rates carry the entire set of information. But from the estimation point of view, the variables, if observed, carry an important piece of information from which we can infer the beliefs of other (representative) agents with higher precision and use them to improve our own policy authority beliefs. Every decision in a DSGE model is, by definition, optimal. And by the construction of the NCC, every decision variable must be present in the estimation. Otherwise, the Kalman filter does not provide optimal estimates. 
The NCC provides a theoretical explanation and support, for example, for the literature on the choice of observable variables (Guerron-Quintana, 2010) or DSGE models in a data-rich environment (Boivin and Giannoni, 2006). GuerronQuintana (2010) addresses the question of "why should we be concerned with the choice of observables?" He experiments with different sets of observables, and on a standard New Keynesian model he shows the effects that their choice has on the parameter estimates and overall model dynamic behavior. At first sight his approach may appear to be data mining, because it is a very data-intensive analysis, but in the light of our argument, Guerron-Quintana's exploration and findings may be justified by natural-condition-of-control arguments.

Our arguments also go in a similar direction as those in Boivin and Giannoni (2006), who propose a framework for exploiting information from large data sets to improve the estimation of DSGE models. In contrast to the data-rich literature we provide a justification of why the use of all the available information in the estimation is a must: this is a dictate of the natural condition of control. Unlike Boivin and Giannoni, who work with empirical relationships, we only use information that can be linked directly to a decision process captured by the model. ${ }^{1}$ In that respect we also use information springing from cross-equation restrictions.

The rest of the paper is structured as follows. In the next section, we review the derivation of the basic Kalman filter equations from an engineering perspective. This will help us to understand the motivation and consequences of the natural condition of control. In the third section, we show how the engineering world maps into the world of dynamic stochastic general equilibrium models. In the fourth section, we illustrate our points on a neoclassical growth model.

\section{State Estimation and Output Prediction}

In engineering, the typical motivation of parameter and/or state estimation is the optimal control problem. The definition of the model is then implied by this task. Consider the discrete-time dynamic system depicted in Figure 1 with the observable/ /measurable input sequence $u_{t}$ and output sequence $y_{t}$ and some hidden variables that can be interpreted as the system parameters $\theta$ or system state $x_{t}$. The input sequence enters in a closed loop in which the control decision is based on the system states estimates.

\subsection{Optimal Control Problem}

Let the sequence of input and output data observed in the time interval from $t_{1}$ up to $t_{2}$ be $D_{t 2}^{t 1}=\left\{u_{t 1}, y_{t 1}, \ldots, u_{t 2}, y_{t 2}\right\}$. If the initial time $t_{1}=1$, it can be omitted, i.e., $D_{1}^{t}=D^{t}$.

Suppose we have observed the input-output sequence up to time $t$ and are looking for optimal control on the $T$ step prediction horizon with optimality criterion $\min E\left\{J\left(D_{t+1}^{T+1}\right) \mid D^{t}\right\}$. This optimization problem requires the joint probability den-

\footnotetext{
${ }^{1}$ From the logic of NCC, the methodology proposed in Schorfheide, Sill, and Kryshko (2010) may be viewed as inefficient use of the available information. The off-model variables, if relevant at all, should be used to update the information about the model states (variables, endogenous factors), instead of being treated exogenously.
} 
Figure 1 Dynamic System Schema (e.g., DSGE Model)

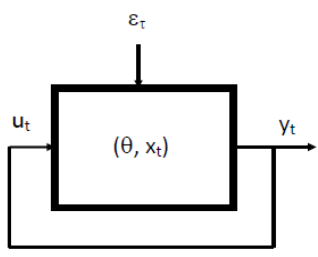

Figure 2 Sampling from a Continuous Process - Logic for the Kalman Filter Timing

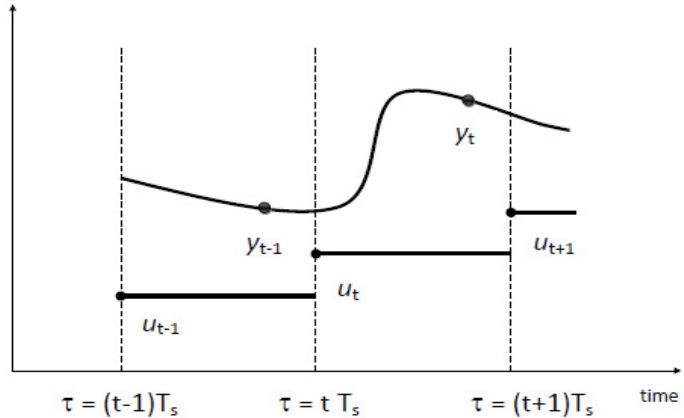

sity function $p\left(D_{t+1}^{T+1} \mid D^{t}\right)$. Using the chain rule, this c.p.d.f. can be written as

$$
p\left(D_{t+1}^{T+1} \mid D^{t}\right)=p\left(y_{t+T} \mid D_{1}^{t+T+1}, u_{t+T}\right) p\left(u_{t+T} \mid D_{1}^{t+T+1}\right) \times \ldots \times p\left(y_{t+1} \mid D_{1}^{t}, u_{t+1}\right) p\left(u_{t+1} \mid D^{t}\right)
$$

The set of c.p.d.f.s $p\left(y_{\tau} \mid D^{\tau-1}, u_{\tau}\right)$ for $\tau=t+1, \ldots, t+T$ defines the dependence of the system output $y_{\tau}$ on the system history up to time $\tau$-1 and the system input at time $\tau$. These c.p.d.f.s define the model of the system.

The set of c.p.d.f.s $p\left(u_{\tau} \mid D^{\tau-1}\right)$ for $\tau=t+1, \ldots, t+T$ is a general description of the law by which the input $u_{\tau}$ is generated. We will call this set of c.p.d.f.s the control law. Note the information delay in the control law; while the input $u_{\tau}$ is applied to the system to generate its output in the $\tau$-th period, the output $y_{\tau}$ is not available to calculate the control law $u_{\tau}$.

In engineering applications, it is typically assumed that a continuous process is observed at regular intervals $\tau=t T_{s}$ with sampling period $T_{s}$ and the input is constant during the sampling period, i.e., $u_{\tau}=u_{t}$ for $t T_{s} \leq \tau<(t+1) T_{s}$. Figure 2 captures the sampling graphically.

\subsection{State Estimation}

If there exists a hidden (latent) variable $x_{t}$ of fixed dimension such that

$$
p\left(x_{t+1}, y_{t} \mid D^{t-1}, x_{t}, u_{\tau}\right)=p\left(x_{t+1}, y_{t} \mid x_{t}, u_{t}\right)
$$

it is called the state of the system. The state of the system $x_{t}$ constrains all the in- 
formation about the system history that is relevant to predict the values $\left\{x_{t+1}, y_{t}\right\}$. Using the state definition above, the output model can be obtained as a marginal distribution

$$
p\left(y_{t} \mid x_{t}, u_{\tau}\right)=\int p\left(x_{t+1}, y_{t} \mid x_{t}, u_{t}\right) d x_{t+1}
$$

and the state transition model as a conditioned distribution

$$
p\left(x_{t+1} \mid x_{t}, u_{t}, y_{t}\right)=\frac{p\left(x_{t+1}, y_{t} \mid x_{t}, u_{t}\right)}{p\left(y_{t} \mid x_{t}, u_{t}\right)}
$$

This reflects the fact that for the prediction of state $x_{t+1}$, the information about the output in the $t$-th period is available and should be incorporated into the optimal prediction (see the sampling scheme in Figure 2). To calculate the output prediction

$$
p\left(y_{t} \mid D^{t-1}, u_{\tau}\right)=\int p\left(y_{t} \mid x_{t}, u_{t}\right) p\left(x_{t} \mid D^{t-1}, u_{t}\right) d x_{t}
$$

information about the state given by the c.p.d.f. $p\left(x_{t} \mid D^{t-1}, u_{t}\right)$ is required at each step of the recursion. That is the point at which the NCC comes into play.

Suppose the information about the state $p\left(x_{t} \mid D^{t-1}\right)$ based on the data up to time $t-1$ is available. This information can be updated after a new input-output observation $\left\{u_{t}, y_{t}\right\}$ has been obtained using the Bayes formula

$$
p\left(x_{t} \mid D^{t}\right)=\frac{p\left(y_{t} \mid D^{t-1}, x_{t}, u_{t}\right) p\left(x_{t} \mid D^{t-1}, u_{t}\right)}{p\left(y_{t} \mid D^{t-1}, u_{t}\right)}=\frac{p\left(y_{t} \mid x_{t}, u_{t}\right)}{p\left(y_{t} \mid D^{t-1}, u_{t}\right)} p\left(x_{t} \mid D^{t-1}\right)
$$

where the properties of the state variable and the natural condition of control for the state estimation (Peterka, 1981) $p\left(x_{t} \mid D^{t-1}, u_{t}\right)=p\left(x_{t} \mid D^{t-1}\right)$ are used to get the second term.

The NCC assumption can be deduced not from the properties of the dynamic system itself, but rather from the process of information accumulation. In the technical context, its interpretation is twofold:

1. The condition $p\left(x_{t} \mid D^{t-1}, u_{t}\right)=p\left(x_{t} \mid D^{t-1}\right)$ says that the control variable $u_{t}$ does not provide any additional information about the state of the system $x_{t}$. This assumption is valid, for example, in the framework of observer-based LQG control - incomplete information feedback - with the control variable based on state estimate $u_{t}=f\left(E\left[x_{t} \mid D^{t-1}\right]\right)$. In this case the control variable $u_{t}$ does not provide any additional knowledge than the information contained in the data set $D^{t-1}$.

2. Using the equality

$$
p\left(x_{t} \mid D^{t-1}, u_{t}\right) p\left(u_{t} \mid D^{t-1}\right)=p\left(u_{t} \mid D^{t-1}, x_{t}\right) p\left(x_{t} \mid D^{t-1}\right)
$$

the condition

$$
p\left(x_{t} \mid D^{t-1}, u_{t}\right)=p\left(x_{t} \mid D^{t-1}\right)
$$


implies that also $p\left(u_{t} \mid D^{t-1}, x_{t}\right)=p\left(u_{t} \mid D^{t-1}\right)$. If the state estimation and control is performed by the same person, the system input is based only on the available data and is not modified by the state estimate, which does not provide any "new" information for the calculation of the control law.

\section{General Equilibrium Models}

Now we turn our attention to dynamic stochastic general equilibrium (DSGE) models. Their (log)linear form is

$$
\boldsymbol{\Gamma}_{\mathbf{0}}(\theta) \mathbf{x}_{\mathbf{t}}=\boldsymbol{\Gamma}_{\mathbf{1}}(\theta) E_{t} \mathbf{x}_{\mathbf{t}+\mathbf{1}}+\boldsymbol{\Gamma}_{\mathbf{2}}(\theta) \mathbf{x}_{\mathbf{t}-\mathbf{1}}+\boldsymbol{\Gamma}_{\mathbf{3}}(\theta) \boldsymbol{\varepsilon}_{\mathbf{t}}
$$

where $\mathbf{x}_{\mathbf{t}}$ is an $(n \times 1)$ vector of endogenous variables (log-deviations from their steady state), and $\boldsymbol{\varepsilon}_{\mathrm{t}}$ is a ( $\left.k \times 1\right)$ vector of unobservable exogenous i.i.d. shocks. For notational simplicity, we assume that $n=k$. This assumption is relaxed in the later discussion. $\boldsymbol{\Gamma}_{\mathbf{0}}(\theta), \boldsymbol{\Gamma}_{\mathbf{1}}(\theta), \boldsymbol{\Gamma}_{\mathbf{2}}(\theta)$, and $\boldsymbol{\Gamma}_{\mathbf{3}}(\theta)$ are time invariant matrices of structural parameters. Their elements are functions of deep structural parameters, $\theta$. $E_{t}($.$) is the rational expectation operator conditional on the model M$ and the information available to economic agents at time $t$ - the information matrix is $\Omega_{t} \in\left(\mathbf{x}_{\mathbf{t}}, \mathbf{x}_{\mathbf{t}-1}, \ldots, \mathbf{x}_{\mathbf{0}}, \boldsymbol{\varepsilon}_{\mathbf{t}}, M\right)$. The structural matrices $\boldsymbol{\Gamma}_{\mathbf{0}}(\theta), \boldsymbol{\Gamma}_{\mathbf{1}}(\theta), \boldsymbol{\Gamma}_{\mathbf{2}}(\theta)$, and $\boldsymbol{\Gamma}_{\mathbf{3}}(\theta)$ are such that the model has a unique and stable equilibrium.

Solving for rational expectations $E_{t}($.$) , model (1) has the minimum state re-$ presentation

$$
\mathbf{x}_{\mathbf{t}}=\mathbf{A}(\theta) \mathbf{x}_{\mathbf{t}-\mathbf{1}}+\mathbf{B}(\theta) \boldsymbol{\varepsilon}_{\mathbf{t}}
$$

Equation (2) is the reduced-form model that characterizes the dynamic equilibrium. $\mathbf{A}(\theta)$ and $\mathbf{B}(\theta)$ are functions of $\boldsymbol{\Gamma}$ s and through them they are functions of the deep structural parameters $\theta$.

The model states $\mathbf{x}_{\mathbf{t}}$ are linked to their observed counterparts via the measurement equation

$$
\mathbf{y}_{\mathbf{t}}=\mathbf{C} \mathbf{x}_{\mathbf{t}}
$$

where $\mathbf{y}_{\mathbf{t}}$ is an ( $\left.m \times 1\right)$ vector of observable variables and $\mathbf{C}$ is the $(m \times n)$ (usually identity) matrix that maps the model variables into $\mathbf{y}_{\mathbf{t}}$.

Equations (2) and (3) together establish the state-space representation of the original model (1). ${ }^{2}$ When estimating (2) and (3), the underlying assumptions are: (i) model (1) is a reasonable representation of the world and the decisions taken in it, and (ii) $\mathbf{y}_{\mathbf{t}}$ is the complete information that the outside observer has available to estimate and evaluate $\mathbf{x}_{\mathbf{t}} \cdot$

If an external observer does not use all the available information, the NCC is violated and the Kalman filtering may not be optimal, which sacrifices the estimation

\footnotetext{
${ }^{2}$ It is useful to note at this point that if $n=m$ the state-space model can be written as a finite-order VAR. If $n>m$, the model can be written as an infinite-order VAR. If $n<m$, the state space model is stochastically deficient.
} 
efficiency of the parameters and unobservable variables. We consider two instances in which the NCC is violated.

\subsection{Learning from Others}

If any additional information about the system state is available for calculating the control law, the standard Kalman filter is not optimal from the Bayesian inference/information accumulation point of view. That is why some applications in the economic literature may not fully comply with the NCC assumption: typically, in a multi-agent environment where individual agents operate based on different information content, the control action of one agent may provide additional information to the remaining agents, i.e., $p\left(x_{t} \mid D^{t-1}, u_{t}\right) \neq p\left(x_{t} \mid D^{t-1}\right)$. If this additional information is not used to evaluate their optimal control strategy, their behavior is not optimal from the Bayesian inference/information accumulation point of view.

As an example, consider a statistician observing a linear system controlled by (complete information) state feedback. Then his (noisy) observation of the controlled variable $u_{t}=-K x_{t}+e_{t}^{u}$ provides significant information about the state.

If the statistician knows the control law $K$, interpreting the control variable $u_{t}$ as an additional observation defined by the c.p.d.f. $p\left(u_{t} \mid x_{t}\right)=p_{e^{u}}\left(u_{t}+K x_{t}\right)$ in parallel to the observed outputs $y_{t}=C x_{t}+D u_{t}+e_{t}^{y}$ defined by $p\left(y_{t} \mid x_{t}, u_{t}\right)=p_{e^{y}}\left(y_{t}-C x_{t}-D u_{t}\right)$, the optimal data update step of the state estimation process (Kalman filter) should cover the input update step

$$
p\left(x_{t} \mid D^{t-1}, u_{t}\right) \propto p\left(u_{t} \mid x_{t}\right) p\left(x_{t} \mid D^{t-1}\right)
$$

and the output update step

$$
p\left(x_{t} \mid D^{t-1}, u_{t}\right) p\left(x_{t} \mid D^{t-1}, u_{t}, y_{t}\right) \propto p\left(y_{t} \mid x_{t}, u_{t}\right) p\left(x_{t} \mid D^{t-1}, u_{t}\right)
$$

If the statistician does not know the control law $K$, he is not able to incorporate this information into the state estimation process. However, if he knows that the NCC is not satisfied ${ }^{3}$ and he is sure that the observed control variable $u_{t}$ provides additional information about the state $x_{t}$, he may try to recover this information. One of his options is to adapt his behavior based on estimation of the control law $K$ as an unknown parameter of the observation model $p\left(u_{t} \mid x_{t}, K\right)$.

The NCC adds an additional dimension to adaptive learning. The basic Kalman filter algorithm already utilizes the information from one's own past prediction errors. In contrast to learning from one's own errors, the violation of the NCC calls for learning from the decisions and errors of others.

\subsection{Unobserved-Observed Variables}

The state equation (2) can be viewed as a model of control in a closed loop (or full-state control). The latent endogenous variables $x_{t}$ can be split into two parts:

\footnotetext{
${ }^{3}$ Detection of NCC violation may be a separate topic of interest.
} 


$$
\boldsymbol{\Gamma}_{\mathbf{0}}(\theta) \mathbf{x}_{\mathbf{t}}=\boldsymbol{\Gamma}_{\mathbf{0}}\left(\theta_{1}\right) \mathbf{x}_{\mathbf{t}}+\mathbf{u}_{\mathbf{t}}
$$

$\mathbf{u}_{\mathbf{t}}=\boldsymbol{\Gamma}_{\mathbf{0}}\left(\theta_{2}\right) \mathbf{x}_{\mathbf{t}}$ is the cumulative effect of the structural parameters $\boldsymbol{\Gamma}_{\mathbf{0}}\left(\theta_{2}\right)$.

If there is $\mathbf{u}_{\mathbf{t}}$ that is observed, we have to extend the observation equation (3) to inform the estimates of $\theta$ and $\mathbf{x}_{\mathbf{t}}$, similarly as in the previous subsection. Therefore, we augment measurement equation (3) to the form of

$$
\begin{gathered}
\mathbf{x}_{\mathbf{t}}=\mathbf{A}(\theta) \mathbf{x}_{\mathbf{t}-\mathbf{1}}+\mathbf{B}(\theta) \boldsymbol{\varepsilon}_{\mathbf{t}} \\
{\left[\begin{array}{l}
\mathbf{u}_{\mathbf{t}} \\
\mathbf{y}_{\mathbf{t}}
\end{array}\right]=\left[\begin{array}{cc}
\boldsymbol{\Gamma}_{\mathbf{0}}\left(\theta_{2}\right) & 0 \\
0 & \mathbf{C A}(\theta)
\end{array}\right] \mathbf{x}_{\mathbf{t}-1}+\left[\begin{array}{cc}
I & 0 \\
0 & \mathbf{C B}(\theta)
\end{array}\right]\left[\begin{array}{l}
\boldsymbol{\varepsilon}_{\mathbf{t}}^{\mathbf{u}} \\
\boldsymbol{\varepsilon}_{\mathbf{t}}
\end{array}\right]}
\end{gathered}
$$

Income tax, consumption tax, or capital (property) tax revenues can serve as an example of a variable $\boldsymbol{u}_{\boldsymbol{t}}$ in DSGE models. These variables almost never appear explicitly in DSGE models. They are determined by passive fiscal policy. Tax rates affect the dynamics indirectly via resource allocation, but tax revenues per se never explicitly appear in the minimum-state representation because they do not bring any additional information about the aggregate dynamics. From the estimation perspective, including observations on tax revenues may be important. It is very often the case that some of the variables in the minimum-state model do not have an observable counterpart (e.g. the capital stock or the output gap). Then, to minimize the uncertainty around their estimates, any information on capital tax revenues is very useful because it is structurally linked to the unobserved capital stock, which helps one to effectively infer its level. ${ }^{4}$

\section{Illustration}

This section illustrates our point that using all the available information may improve models' adaptability and estimation efficiency. We use a simple real business cycle (RBC) model to generate artificial data on private consumption, hours worked, investment, consumption tax receipts, and disposable income, which form our set of available information. First, we assume that an econometrician (observer) uses only two series out of the complete information set. Next, we gradually expand the set that the econometrician uses. Then a similar exercise is repeated on actual data.

The RBC model comprises two sets of agents - households and firms. Households maximize their expected lifetime welfare

$$
E_{0} \sum_{t=0}^{\infty} \beta^{t}\left[\frac{\left(C_{t}+H_{t}\right)^{\sigma}}{1-\sigma}+\xi \log \left(1-L_{t}\right)\right]
$$

subject to the budget constraint

$$
w_{t} L_{t}+\left(1+r_{t}-\delta\right) K_{t-1}+T_{t}=\left(1-\tau_{c}\right) C_{t}+K_{t}
$$

\footnotetext{
${ }^{4}$ Similarly, the demand for money may add additional power to output gap forecasts. It does not carry any additional information about the inflation rate and output gap, because money demand follows the demand on those factors. But exactly the very same reason dictates the inclusion of money demand among the observable variables if the output gap is unobservable, because observed money demand can help to pin down more precisely the unobserved output gap.
} 
The parameter $\sigma>0$ is the measure of the household's risk aversion, and the parameter $\beta \in(0,1)$ is the time discount factor. The household's welfare derives from consumption $C_{t}$ and leisure $1-L_{t}$. The level of consumption is supported by the consumption habit $H_{t}$, which depends on past consumption and an i.i.d. habit shock $H_{t}=\varphi C_{t-1} e^{\varepsilon_{t}}$, with $\varphi \in(0,1)$ and $\varepsilon_{t} \sim N\left(0, \sigma_{c}^{2}\right)$. Time spent working $L_{t}$ causes disutility but is compensated by the hourly real wage $w_{t}$. Consumption is taxed by the government at the rate of $\tau_{c} \in(0,1)$. The household is the only owner of physical capital $K_{t}$ in the economy, which is, together with labor, a factor of production. Firms rent the capital and pay households interest $r_{t}$ in return, but the physical capital depreciates over time at the rate $\delta \in(0,1)$. The household also receives lump-sum transfers $T_{t}$ from the government, which operates on a balanced budget.

Firms maximize their profits $\Pi_{t}=Y_{t}-r_{t} K_{t-1}-w_{t} L_{t}$ by optimally hiring labor and capital to produce the consumption good $Y_{t}$ using Cobb-Douglas technology: $Y_{t}=A_{t} K_{t-1}^{\alpha} L_{t}^{1-\alpha} . A_{t}$ is the total factor productivity and follows a log-linear $\operatorname{AR}(1)$ process: $\log A_{t}=\rho \log A_{t-1}+\varepsilon_{t}^{A}$. The exogenous shock $\varepsilon_{t}^{A} \sim N\left(0, \sigma_{A}^{2}\right)$ and we interpret it as the productivity shocks. $\alpha \in(0,1)$ is the share of capital in production.

In equilibrium, all (labor, capital, and consumption goods) markets clear. The dynamic equilibrium is characterized by the Euler equation for consumption, labor demand, the resource constraint, and the exogenous supply of technology.

$$
\begin{aligned}
\left(\frac{C_{t}+\varphi C_{t-1} e^{\varepsilon_{t}}}{E_{t} C_{t+1}+\varphi C_{t}}\right)^{-\sigma} & =\beta\left(1-\delta-\alpha A_{t} K_{t-1}^{\alpha-1} L_{t}^{1-\alpha}\right) \\
\xi \frac{1-L_{t}}{C_{t}^{\sigma}} & =\left(\frac{1-\tau_{c}}{1-\alpha}\right) \frac{L_{t}^{\alpha}}{A_{t} K_{t-1}^{\alpha}} \\
A_{t} K_{t-1}^{\alpha-1} L_{t}^{1-\alpha} & =C_{t}+K_{t}-(1-\delta) K_{t-1} \\
\log A_{t} & =\rho \log A_{t-1}+\varepsilon_{t}^{A}
\end{aligned}
$$

We use the following values to parameterize the equilibrium: $\alpha=0.60$, $\beta=0.97, \xi=1, \sigma=3, \tau_{c}=0.2, \delta=0.01, \varphi=0.5, \rho=0.9, \sigma_{A}=\sigma_{c}=0.01$.

The model is solved using the methodology proposed by King, Plosser, and Rebelo (1988). First, the model steady state is computed. Second, (6)-(9) are log-linearized around the steady state, and we obtain the model in the form of (1). Finally, the log-linear model is solved for the rational expectations $E_{t}($.$) . The result is$ the state equation (2), in which there are four endogenous variables $\left[C_{t}, K_{t}, L_{t}, A_{t}\right]$, three of which are truly state variables, ${ }^{5}$ and there are two structural shocks $\left[\varepsilon_{t}, \varepsilon_{t}^{A}\right]$.

${ }^{5}\left[C_{t-1}, K_{t-1}, A_{t-1}\right]$ are the truly state variables. They form the minimum-state-variable solution to the DSGE model. The equilibrium level of $L_{t}$ follows from the marginal rate of substitution between work and consumption, which is an intratemporal/static relationship. 
Having the model, we simulate the set of available information. The set consists of the measures of private consumption $\bar{C}_{t}$ hours worked $\bar{L}_{t}$, gross private investment $\bar{I}_{t}$, sales tax receipts $\bar{T}_{t}$, and disposable income $\bar{D} \bar{I}_{t}$. We assume that the measures of these variables are published by a statistics office. We do not measure any direct counterparts of the physical capital stock and the total factor productivity. Those variables remain latent states in the exercise.

The first two observed variables are direct counterparts of the state variables $\bar{C}_{t}$ and $\bar{L}_{t}$. The other three are definitions implicitly included in (6)-(9). They are functions of the model's endogenous variables. Gross private investment is defined as $\bar{I}_{t}=K_{t}-(1-\delta) K_{t-1}$, consumption tax receipts are $\bar{T}_{t}=\tau_{c} C_{t}$, and disposable income is equal to equilibrium production $\bar{D} \bar{I}_{t}=A_{t} K_{t-1}^{\alpha-1} L_{t}^{1-\alpha}$. All the variables are measured with an error that is i.i.d.

\subsection{Monte Carlo Experiment}

We assume that an econometrician (observer) who wants to estimate the model (6)-(9) decides to use the information contained only in the measures of consumption $\bar{C}_{t}$ and hours worked $\bar{L}_{t}$. He knows the structural model and its parametrization, and he wants to estimate the latent states $\bar{K}_{t}$ and $\bar{A}_{t}$.

The assumptions of this experiment resemble a setup common in economic applications. The econometrician knows the available data, but he decides to use the measures that are naturally the closest to the model variables. Because there are no direct counterparts of $\bar{K}_{t}$ or $\bar{A}_{t}$ in his database, he treats them as latent and estimates them. The structural parameters are known to him and thus the econometrician only seeks $\bar{K}_{t}$ and $\bar{A}_{t}$. The Kalman filter can deliver their optimal estimates.

The estimation results of this experiment are plotted in Figure 3. The solid lines are the actual (simulated and known to us) series of $\bar{K}_{t}$ (top panel) and $\bar{A}_{t}$ (lower panel). The widest shadow interval corresponds in both panels to the uncertainty of the estimates.

The econometrician's choice of observable variables results in a loss of efficiency and consistency. We see that very often the estimate of capital or technology is indistinguishable from zero. The confidence intervals are wide but at the same time may not include the actual series.

In the next step, the econometrician exploits the available information a bit more. He realizes that the statistics office provides more data that is structurally linked to his model and can help to inform his estimates. The light intervals in Figure 3 show the gain in efficiency and consistency when disposable income is introduced into the set of observable variables; data on investment are similarly informative. The confidence intervals for the capital stock and technology estimates shrink. The estimates become statistically significant and more closely match the actual underlying trajectory of the latent variables. The tax revenues contribute only marginally to the accuracy of the estimates. 


\section{Figure 3 Estimates of Capital Stock and Labor Productivity (MC Experiment)}

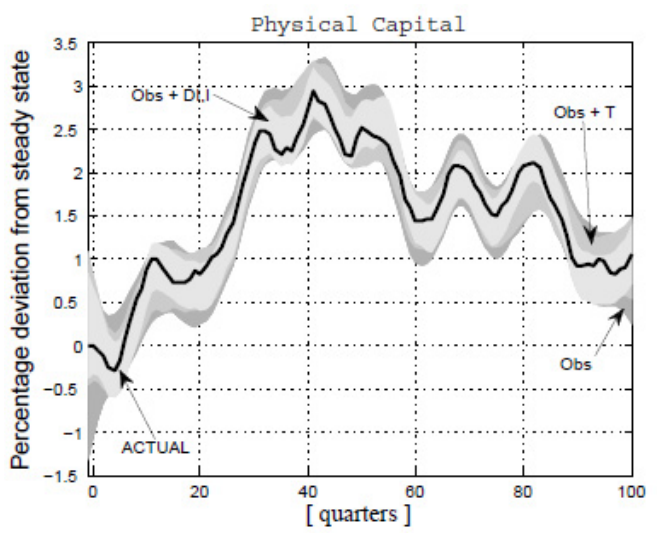

Notes: The graph presents the results of a Monte Carlo experiment with model (6)-(9) as parameterized in the text. The shocks are drawn from i.i.d.s. In both panels, the solid line is the actual series (capital stock - top panel; technology - lower panel). The shaded bands around the actual series are the estimated 2std (smoothed) confidence intervals conditioned on a set of observables. The baseline information set (Obs) - consumption and hours worked observed - and the baseline set extended to include consumption tax receipts (Obs+T) yield the two widest confidence intervals. The baseline set extended to include disposable income $(\mathrm{Obs}+\mathrm{DI})$ or investment $(\mathrm{Obs}+\mathrm{l})$ provides the narrowest confidence intervals and pins down the level of the actual states very precisely.

This Monte Carlo experiment also illustrates why violation of the NCC differs from the problem of model misspecification and is therefore hard to detect. Unlike model misspecification, violation of the NCC does not have a clear manifestation in the estimation outcome, e.g. the shock estimates are not i.i.d. The Kalman filter optimally processes data information. It always provides optimal estimates of all latent variables. For example, all the confidence intervals plotted in Figure 3 are based on a well specified model and well behaved estimates, so more of them can be dismissed as inefficient or inconsistent. And still some of the data sets well outperform others in the estimation.

In practice, it is difficult to make ad hoc claims about which of the available data add the most efficient information, but the model structure may help with the inference. Prior to any estimation we can evaluate the Fisher information matrix. We may infer how much new information we can expect to obtain when asking a particular set of data. It is a coherent way to summarize and analyze the information content presented, for example, in Figure 3. The Fisher information matrix can help us to prioritize among the variables we are thinking of selecting from the set of available information, which may be particularly helpful if we happen to have a constraint on the available computation power. In contrast to the selection criteria proposed in Guerron-Quintana (2010), the analysis of the Fisher information matrix appears to be a cleaner way to prioritize among the observable variables, because it does not require any prior data information.

\subsection{Estimated Model}

Now we repeat the above experiments with actual US data. In contrast to the prior analysis, we will see that, empirically, consumption and hours worked suf- 
ficiently inform the estimates of capital and technology. Adding the observations on fixed private investment does not add to the efficiency very much.

The data used in this section are taken from the Federal Reserve Economic Data managed by the Federal Reserve Bank of St. Louis. ${ }^{6}$ We use the annual series of real personal consumption expenditures (mnemonic: PCECCA96), the annual series of real private fixed investment (mnemonic: FPICA), and the annualized series of total hours worked, which is the product of the monthly seasonally adjusted series of average weekly hours worked in private industries (mnemonic: AWHNONAG) and total non-farm payrolls (mnemonic: PAYEMS). Per capita terms are taken with respect to the total civilian labor force (mnemonic: CLF16OV). We consider two time spans. The first one is relevant to consumption and spans from 1949 to 2009. The second is for hours worked and investment, which we observe from 1965 and 1967 , respectively, to 2009 . The model is estimated on the relevant samples between 1950 and 2009.

Because model (6)-(8) has no nominal rigidities, we treat it as a growth model and estimate it at an annual frequency. Because of the non-stationary nature of the actual data, we modify the technological process to include a stochastic trend. Instead of (9) we now assume that the technology $A_{t}$ is labor augmenting and follows the first-difference stationary process with drift:

$$
\Delta \log A_{t}=(1-\rho) \Delta \bar{A}+\rho \Delta \log A_{t-1}+\varepsilon_{t}^{A}
$$

$\Delta \bar{A}>0$ is the drift term, which sets the economy on an exogenous but balanced growth path. Both capital and consumption grow at that rate in the long run.

The transitory parameters $\{\Phi, \rho\}$ and the variances $\left\{\varepsilon_{t}^{c}, \varepsilon_{t}^{a}\right\}$ are estimated using the maximum likelihood method. ${ }^{7}$ The other parameters are kept fixed at their parameterized values mentioned above. We will not report their estimates and instead we again focus on the estimates of the capital stock $K_{t}$ and labor-augmenting technology $A_{t}$.

Figure 4 summarizes the basic results. In the top two panels, we compare the confidence intervals for the smoothed estimates of $K_{t}$ and $A_{t}$. The panels show the relative efficiency of the capital stock estimate (top left) and labor-augmenting technology estimate (top right) when (i) the information on the growth of consumption and hours worked is used (model 1), and (ii) that information is extended to include investment growth (model 2). The shaded areas are then computed as $100\left[\operatorname{std}\left(X_{t, \text { model } 1}\right) / \operatorname{std}\left({ }_{t, \text { model } 2}\right)-1\right]$. Positive values mean that model 2 (the model with more information) outperforms model 1 (the model with less information).

The model with more information (Figure 4, model 2) helps to improve the estimate of labor-augmenting technology (right panel). Early in the sample the relative efficiency of model 2 is seven times higher than that of the model with less information. The relative advantage of model 2 gradually diminishes as model 1 adapts and as its efficiency improves over time. By 2010, the relative efficiency of model 2 is only two times better than that of model 1 .

\footnotetext{
${ }^{6}$ See: http://research.stlouisfed.org/fred2/.

${ }^{7}$ The parameters are estimated to allow for a better data match. This does not represent a fundamental change to the setup of the experiment.
} 
Figure 4 Estimates of the Capital Stock and Labor Productivity (US data)
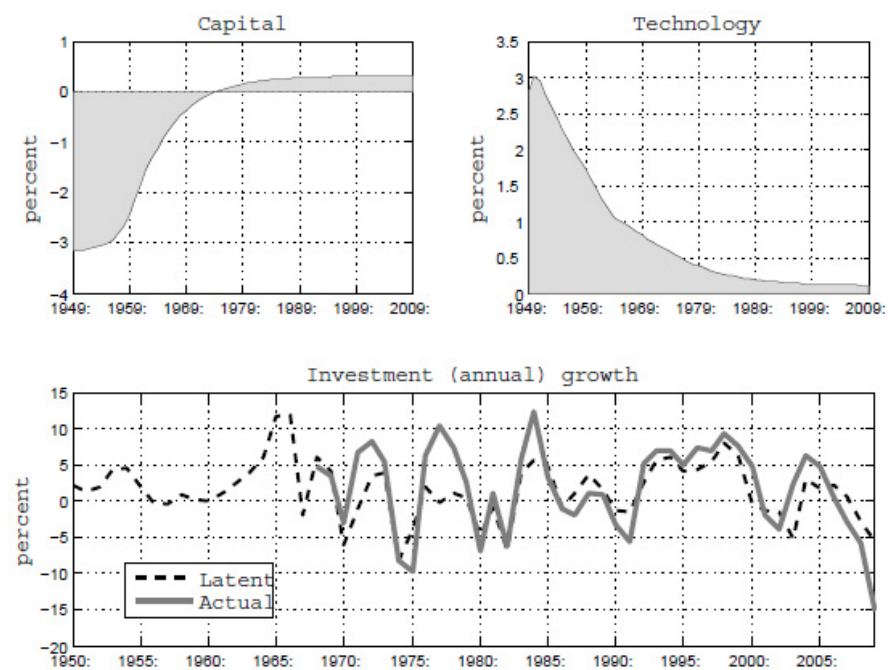

Notes: The top two panels show the relative efficiency of the capital stock estimate (top left) and the labor-augmenting technology estimate (top right) when (i) the information on the growth of consumption and hours worked is used (model 1), and (ii) that information is extended to include investment growth (model 2). The shaded areas are computed as $100\left[s t d\left(X_{t, \text { model } 1}\right) / s t d(t\right.$, mode/2 $\left.)-1\right]$.

We recall that the estimates are smoothed estimates, that is, the early estimates of $A_{t}$ are based on the complete information set available at time $T$. That is why the model with more information performs better over the whole sample even if the extra information in the form of investment growth comes in after 1967.

In the case of the capital stock (Figure 4, left panel), model 2 starts to outperform model 1 shortly before 1971, when the new information from hours worked and investment begins to feed in. Early in the sample, the model with less information performs almost seven times better than model 2. This is the price of the improved estimate for the technological process in this time period. After 1971, the model with more information again clearly outperforms model 1 , delivering estimates of the capital stock that are twice as efficient as in model 1.

One may wonder why the observations on investment do not provide even higher gains in efficiency. The model's good (in-sample) predictive power for investment provides an explanation. The graph in the bottom panel of Figure 4 compares the model-implied investment (when treated as latent in model 1) with the actually realized (observed) data. Clearly, the data on private consumption expenditures and hours worked by themselves contain enough information about investment and thus the capital stock and also technology.

\section{Final Remarks}

We reviewed the basic derivation of Kalman filter equations with a focus on the role of the natural condition of control. We were interested in what this condition implied for the estimation of DSGE models used in economics. We provided a theoretically consistent justification for the use of all available (observable) information 
that can be structurally linked to the model. Under the assumption of information pooling, we illustrated that this leads to significantly improved estimate efficiency.

The NCC can provide an alternative structural perspective for DSGE model developers. The model may be well specified, but the NCC still can be violated. This is because the condition deals not with the model structure per se, but rather with the flow of information in it.

In future work we would like to look at possible avenues for formally testing the NCC, which can be used for empirically assessing endogenous decision rules. DSGE models consist of optimal decision (control) rules, so each equation can be subject to testing. Another possible avenue for research is to relax the assumption of information pooling and look at the case of an agent with significant market power and private information. If the NCC holds, the remaining market players can try to infer the private information encoded in the decisions of the dominant player and adapt to it.

\section{REFERENCES}

Boivin J, Giannoni M (2006): DSGE Models in a Data-Rich Environment. NBER Working Paper, no.12772.

King RG, Plosser Ch, Rebelo ST (1988): Production, growth and business cycles: I. The basic neoclassical model. Journal of Monetary Economics, 21(2-3):195-232.

Peterka V (1981): Bayesian Approach to System Identification. In: Eykhoff P (Ed.): Trends and Progress in System Identification. Pergamon Press, Oxford. Also available at http://moodle.utia.cas.cz/moodledata/4/peterka.pdf

Schorfheide F, Sill K, Kryshko M (2010): DSGE Model-Based Forecasting of Non-Modeled Variables. International Journal of Forecasting, 26(2):348-373.

Guerron-Quintana PA (2010): What You Match Does Matter: The Effects of Data on DSGE Estimation. Journal of Applied Econometrics, 25(5):774-804. 